\title{
Nonsmooth Lur'e Dynamical Systems in Hilbert Spaces
}

\author{
Samir Adly ${ }^{1}$ Abderrahim Hantoute - Ba Khiet Le $^{2}$
}

Received: 12 December 2014 / Accepted: 9 June 2015 / Published online: 4 July 2015

(C) Springer Science+Business Media Dordrecht 2015

\begin{abstract}
In this paper, we study the well-posedness and stability analysis of set-valued Lur'e dynamical systems in infinite-dimensional Hilbert spaces. The existence and uniqueness results are established under the so-called passivity condition. Our approach uses a regularization procedure for the term involving the maximal monotone operator. The Lyapunov stability as well as the invariance properties are considered in detail. In addition, we give some sufficient conditions ensuring the robust stability of the system in finitedimensional spaces. The theoretical developments are illustrated by means of two examples dealing with nonregular electrical circuits and an other one in partial differential equations. Our methodology is based on tools from set-valued and variational analysis.
\end{abstract}

Keywords Nonsmooth Lur'e dynamical systems · Well-posedness · Stability and robustness $\cdot$ Maximal monotone operators

Mathematics Subject Classifications (2010) $37 \mathrm{~J} 25 \cdot$ 46N10 • 49J53 $\cdot 37 \mathrm{~B} 25$

Dedicated to professor Lionel Thibault on the occasion of his birthday.

Samir Adly

samir.adly@unilim.fr

Abderrahim Hantoute

ahantoute@dim.uchile.cl

Ba Khiet Le

lkhiet@dim.uchile.cl

1 XLIM UMR-CNRS 7252, Université de Limoges, 87060 Limoges, France

2 Centro de Modelamiento Matemático (CMM), Universidad de Chile, Santiago, Chile 


\section{Introduction}

The Lur'e dynamical systems consist of a coupling of a negative feedback interconnection of a linear time-invariant forward path $(A, B, C, D)$ and a static nonlinear feedback $\mathcal{F}$. Such systems are of frequent use in control theory and have many applications in engineering and applied mathematics (see, e.g., [1] and references therein). For instance, many problems in electrical and mechanical engineering can be formulated by set-valued Lur'e dynamical systems. This is the case, for example, in nonregular electrical circuits, in designing state observers or in control of systems subjected to dry friction [2, 3]. More recently, Lur'e dynamical systems with set-valued static feedback part have been used and studied in [2-7]. It is also known that other mathematical models used to study non-smooth dynamical systems (relay systems, evolution variational inequalities, projected dynamical systems, complementarity systems...) can be formulated into Lur'e dynamical systems with a set-valued feedback nonlinearity [6, 8-11].

This paper considers the set-valued Lur'e dynamical systems in infinite dimensional Hilbert spaces. Let $\mathcal{H}_{1}, \mathcal{H}_{2}$ be two Hilbert spaces, and consider bounded linear operators $A: \mathcal{H}_{1} \rightarrow \mathcal{H}_{1}, B: \mathcal{H}_{2} \rightarrow \mathcal{H}_{1}, C: \mathcal{H}_{1} \rightarrow \mathcal{H}_{2}$ and $D: \mathcal{H}_{2} \rightarrow \mathcal{H}_{2}$. We also consider a set-valued map $\mathcal{F}: \mathcal{H}_{2} \rightrightarrows \mathcal{H}_{2}$. Then, given an initial point $x_{0} \in \mathcal{H}_{1}$, the problem consists of finding an absolutely continuous function $x(\cdot)$ defined on $[0,+\infty)$ such that

$$
(\mathcal{S})\left\{\begin{array}{l}
\dot{x}(t)=A x(t)+B \lambda(t) \text { a.e. } t \in[0,+\infty) ; \\
y(t)=C x(t)+D \lambda(t), \\
y(t) \in \mathcal{F}(-\lambda(t)), t \geq 0 ; \\
x(0)=x_{0},
\end{array}\right.
$$

where $\lambda, y: \mathbb{R}_{+} \rightarrow \mathcal{H}_{2}$ are two connected unknown mappings. It is possible to include perturbation with locally integrable external force $f(\cdot)$ and/or a nonlinear Lipschitz continuous map instead of the linear operator $A$ but, for simplicity, we restrict ourselves to system $(\mathcal{S})$. To the best of our knowledge, such system was firstly introduced and analysed in a special case in [5].

In order to illustrate our systems $(\mathcal{S})$, we give the following classical scheme in Fig. 1 (see, e.g., [6]). Here $A, B, C, D$ are referred to as the state, the input, the output, and the feedthrough operators, respectively. Most of the previous works concern the finitedimensional case where the matrix $D=0$ [4] or $D \neq 0$ and $\mathcal{F}$ is a maximal monotone operator $[2,6,7]$. The consideration of nonzero matrix $D$ makes the analysis of the system more difficult. If $\mathcal{F}$ coincides with the normal cone of $\mathbb{R}_{+}^{n}$ (i.e. $\mathcal{F}=N_{\mathbb{R}_{+}^{n}}$ ), then $\operatorname{system}(\mathcal{S})$ reduces to the well-known linear complementarity systems largely studied in the literature $[3,12]$. In [6], the authors studied the well-posedness, stability and invariance properties of

Fig. 1 Set-valued Lur'e block diagram

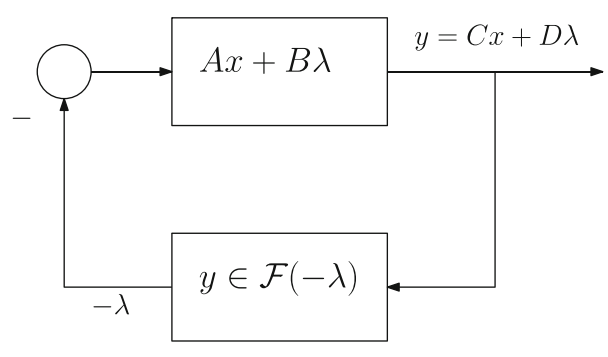


system $(\mathcal{S})$, where $\mathcal{F}$ is the inverse of the subdifferential mapping of a given proper convex and lower semicontinuous function, or, equivalently, the subdifferential mapping of its Fenchel conjugate. The well-posedness is improved in [7] for a general maximal monotone operator by using the passivity of the linear systems. It is worth noting that in the case $D=0$, the monotonicity of set-valued map $\mathcal{F}$ is not necessary; in fact, only the local hypo-monotonicity of $\mathcal{F}$ is sufficient [4].

In the present paper, nonzero operator $D$ is allowed. The well-posedness and the stability analysis, including finite-time stability, of $(\mathcal{S})$ are investigated in the general setting of infinite-dimensional Hilbert spaces. When the underlying spaces are finite-dimensional, we generalize a version of Krasovskii-LaSalle invariance principle, study the asymptotic behaviour of solutions and investigate the attractive region. Furthermore, we give a condition that guarantees the attractivity of the set of equilibria. We also give sufficient conditions ensuring the robust stability of $(\mathcal{S})$ (see, e.g., [13]).

The paper is organized as follows: in Section 2, we recall some results in set-valued analysis and extend some definitions of control theory to the infinite-dimensional setting. The well-posedness and stability analysis of the system $(\mathcal{S})$ are studied in detail in Section 3 and Section 4, respectively. Section 5 is dedicated to the analysis of the robust stability of the Lur'e dynamical system $(\mathcal{S})$. In Section 6, we present two examples in nonregular electrical circuits and one example in partial differential equations to support the theoretical results. Some concluding remarks are given in Section 7.

\section{Notation and Mathematical Backgrounds}

Let us first introduce some notation that will be used in the sequel. Denote by $\langle\cdot, \cdot\rangle,\|\cdot\|$ the scalar product and the corresponding norm in a Hilbert space $\mathcal{H} ; I$ the identity operator, $\mathbb{B}_{\mathcal{H}}$ the closed unit ball in $\mathcal{H}$. The interior of a given set $S \subset \mathcal{H}$ is denoted by $\operatorname{int}(S)$. The domain, the range and the graph of a set-valued mapping $A: \mathcal{H} \rightrightarrows \mathcal{H}$ are defined respectively by

$$
\operatorname{dom}(A)=\{x \in \mathcal{H}: A(x) \neq \emptyset\}, \quad \operatorname{rge}(A)=\bigcup_{x \in \mathcal{H}} A(x)
$$

and

$$
\operatorname{gph}(A)=\{(x, y): x \in \mathcal{H}, y \in A(x)\} .
$$

The inverse operator $A^{-1}$ is defined by

$$
x \in A^{-1}(y) \Leftrightarrow y \in A(x) \text { for all } x, y \in \mathcal{H} .
$$

Obviously, one has $\operatorname{dom}\left(A^{-1}\right)=\operatorname{rge}(A)$ and $\operatorname{rge}\left(A^{-1}\right)=\operatorname{dom}(A)$. The operator $A$ is said to be monotone if for all $x, y \in \mathcal{H}, x^{*} \in A(x), y^{*} \in A(y)$, we have $\left\langle x^{*}-y^{*}, x-\right.$ $y\rangle \geq 0$. The operator $A$ is said to be maximal monotone if there is no monotone set-valued mapping $B$ such that $\operatorname{gph}(A)$ is contained strictly in $\operatorname{gph}(B)$. Let $A, B: \mathcal{H} \rightrightarrows \mathcal{H}$ be two maximal monotone operators on $\mathcal{H}$ such that $\operatorname{dom}(A) \cap \operatorname{int}(\operatorname{dom}(B)) \neq \emptyset$, then $A+B$ is maximal monotone. Particularly, we note that if $B: \mathcal{H} \rightarrow \mathcal{H}$ is single-valued, monotone and continuous then $A+B$ is maximal monotone (see e.g. [14]). In the following, we summarize some well-known results concerning maximal monotone operators that will be used later.

Proposition 1 ([14-17]) Let $A: \mathcal{H} \rightrightarrows \mathcal{H}$ be a maximal monotone operator and let $\lambda>0$. Then 
1) the resolvent of $A$ defined by $J_{\lambda}^{A}:=(I+\lambda A)^{-1}$ is a non-expansive and single-valued map from $\mathcal{H}$ to $\mathcal{H}$.

2) the Yosida approximation of $A$ defined by $A_{\lambda}:=\frac{1}{\lambda}\left(I-J_{\lambda}^{A}\right)=\left(\lambda I+A^{-1}\right)^{-1}$ satisfies

i) for all $x \in \mathcal{H}, A_{\lambda}(x) \in A\left(J_{\lambda}^{A} x\right)$,

ii) $A_{\lambda}$ is Lipschitz continuous with constant $\frac{1}{\lambda}$ and also maximal monotone.

iii) If $x \in \operatorname{dom}(A)$, then $\left\|A_{\lambda} x\right\| \leq\left\|A^{0} x\right\|$, where $A^{0} x$ is the element of Ax of minimal norm.

3) If $x_{\lambda} \rightarrow x$ and $\left(A_{\lambda} x_{\lambda}\right)_{\lambda>0}$ is bounded as $\lambda \rightarrow 0$, then $x \in$ domA. Moreover, if $y$ is a cluster point of $\left(A_{\lambda} x_{\lambda}\right)_{\lambda>0}$ as $\lambda \rightarrow 0$, then $y \in A(x)$.

Proposition 2 ([14, 16]) Let $A: \mathcal{H} \rightrightarrows \mathcal{H}$ be a maximal monotone operator. Then

1) A is sequentially weak-strong and strong-weak closed.

2) A is locally bounded on int (dom) $A$.

3) For $T>0$, define $\mathcal{A}: L^{2}([0, T] ; \mathcal{H}) \rightrightarrows L^{2}([0, T]$; $\mathcal{H})$ by

$$
y(\cdot) \in \mathcal{A} x(\cdot) \text { iff } y(t) \in A x(t) \text { a.e. } t \in[0, T] .
$$

Then $\mathcal{A}$ is also a maximal monotone operator.

Next let us extend some definitions concerning passive systems which play an essential role in control theory [3], to infinite dimensional spaces.

Definition 1 An operator $P \in \mathcal{L}(\mathcal{H})$ (the set of all linear and bounded operators from $\mathcal{H}$ to $\mathcal{H})$ is said to be

- $\quad$ semi-positive definite (or monotone) iff for all $x \in \mathcal{H}$,

$$
\langle P x, x\rangle \geq 0 \text {. }
$$

- positive definite iff there exists $\alpha>0$ such that for all $x \in \mathcal{H}$,

$$
\langle P x, x\rangle \geq \alpha\|x\|^{2} .
$$

- $\quad$ symmetric iff $P=P^{T}$, i.e., for all $x, y \in \mathcal{H}$, we have $\langle P x, y\rangle=\langle x, P y\rangle$.

Let $A: \mathcal{H}_{1} \rightarrow \mathcal{H}_{1}, B: \mathcal{H}_{2} \rightarrow \mathcal{H}_{1}, C: \mathcal{H}_{1} \rightarrow \mathcal{H}_{2}$ and $D: \mathcal{H}_{2} \rightarrow \mathcal{H}_{2}$ be some given linear and bounded operators where $\mathcal{H}_{1}, \mathcal{H}_{2}$ are Hilbert spaces.

Definition 2 ( $A, B, C, D)$ is said to be passive if there exists a symmetric positive definite operator $P \in \mathcal{L}\left(\mathcal{H}_{1}\right)$ such that for all $x \in \mathcal{H}_{1}, y \in \mathcal{H}_{2}$, we have

$$
\langle P A x, x\rangle+\left\langle\left(P B-C^{T}\right) y, x\right\rangle-\langle D y, y\rangle \leq 0 .
$$

In this case, we say that the system $(A, B, C, D)$ is $P$-passive.

Remark 1 i) In finite dimension, the $P$-passivity of $(A, B, C, D)$ is equivalent to negative semi-definiteness of the matrix

$$
\left(\begin{array}{cc}
P A+A^{T} P & P B-C^{T} \\
B^{T} P-C & -\left(D+D^{T}\right)
\end{array}\right) .
$$


ii) It is easy to see that if the system $(A, B, C, D)$ is $P$-passive then the following dissipation inequality holds

$\frac{1}{2}\left\langle P x\left(t_{1}\right), x\left(t_{1}\right)\right\rangle+\int_{t_{1}}^{t_{2}}\langle y(\tau), \lambda(\tau)\rangle d \tau \geq \frac{1}{2}\left\langle P x\left(t_{2}\right), x\left(t_{2}\right)\right\rangle$ for all $t_{2} \geq t_{1} \geq 0$.

Indeed,

$\frac{d}{d t}\left(\frac{1}{2}\langle P x(t), x(t))-\langle y(t), \lambda(t)\rangle=\langle P x(t), A x(t)+B \lambda(t)\rangle-\langle C x(t)+D \lambda(t), \lambda(t)\rangle \leq 0\right.$.

iii) The inequality in (3) is equivalent to

$$
\langle P A x, x\rangle+\left\langle\left(C^{T}-P B\right) y, x\right\rangle-\langle D y, y\rangle \leq 0 \text { for all } x \in \mathcal{H}_{1}, y \in \mathcal{H}_{2} .
$$

Definition 3 ( $A, B, C, D)$ is said to be strictly $P$-passive if $(A+\varepsilon I, B, C, D)$ is $P$-passive for some $\varepsilon>0$.

Lemma 1 If $(A, B, C, D)$ is $P$-passive, then

(i) $D$ is positive semi-definite.

(ii) For every sequence $\left(y_{n}\right)_{n \geq 1} \subset \mathcal{H}_{2}$ such that $\lim _{n \rightarrow+\infty}\left\langle D y_{n}, y_{n}\right\rangle=0$, we have $\lim _{n \rightarrow+\infty}\left(P B-C^{T}\right) y_{n}=0$.

Proof (i) Taking $x=0$ in (3) for $D$.

(ii) Suppose that $\xi_{n}:=\left(P B-C^{T}\right) y_{n}$ does not converge to 0 . Then there exists $\varepsilon>0$ and a subsequence $\left(\xi_{n_{k}}\right)$ such that $\left\|\xi_{n_{k}}\right\| \geq \varepsilon$ for all $k$. Choose $M>0$ such that $(M-1) \varepsilon-\|P A\| \geq 0$. Take $y=y_{n_{k}}, x=\frac{\xi_{n_{k}}}{M\left\|\xi_{n_{k}}\right\|}$ in (3), then we have

$$
\begin{aligned}
\frac{\left\|\xi_{n_{k}}\right\|}{M} & =\left\langle\left(P B-C^{T}\right) y_{n_{k}}, x_{n_{k}}\right\rangle \leq\left\langle D y_{n_{k}}, y_{n_{k}}\right\rangle-\left\langle P A x_{n_{k}}, x_{n_{k}}\right\rangle \\
& \leq\left\langle D y_{n_{k}}, y_{n_{k}}\right\rangle+\frac{\|P A\|}{M^{2}} .
\end{aligned}
$$

Consequently

$$
\left\langle D y_{n_{k}}, y_{n_{k}}\right\rangle \geq \frac{M\left\|\xi_{n_{k}}\right\|-\|P A\|}{M^{2}} \geq \frac{\varepsilon}{M^{2}} .
$$

Passing to the limit as $k \rightarrow+\infty$ in (5), we get a contradiction.

We end-up this section by recalling a version of Gronwall's inequality (see, e.g., [18]).

Lemma 2 Let $T>0$ be given and $a(\cdot), b(\cdot) \in L^{1}([0, T] ; \mathbb{R})$ with $b(t) \geq 0$ for almost all $t \in[0, T]$. Let an absolutely continuous function $w:[0, T] \rightarrow \mathbb{R}_{+}$satisfy

$$
(1-\alpha) w^{\prime}(t) \leq a(t) w(t)+b(t) w^{\alpha}(t), \text { a.e. } t \in[0, T]
$$

where $0 \leq \alpha<1$. Then for all $t \in[0, T]$, we have

$$
w^{1-\alpha}(t) \leq w^{1-\alpha}(0) \exp \left(\int_{0}^{t} a(\tau) d \tau\right)+\int_{0}^{t} \exp \left(\int_{s}^{t} a(\tau) d \tau\right) b(s) d s .
$$




\section{Existence and Uniqueness of Solutions}

In this section, we study the existence and uniqueness of the solutions of the system $(\mathcal{S})$ by using regularization. From $(1 b)$ and $(1 \mathrm{c})$ of $(\mathcal{S})$, it is easy to compute $\lambda(\cdot)$ in term of $x(\cdot)$ :

$$
\lambda(\cdot) \in-(D+\mathcal{F})^{-1}(C x(\cdot)) .
$$

Therefore, we can rewrite the system $(\mathcal{S})$ in the form of first order differential inclusion as follows

$$
\dot{x}(t) \in A x(t)-B(D+\mathcal{F})^{-1}(C x(t)) \text { a.e. } t \geq 0 .
$$

The domain of the right-hand side of $(8)$ is $C^{-1}\left(\operatorname{dom}(D+\mathcal{F})^{-1}\right)=C^{-1}(\operatorname{rge}(D+\mathcal{F}))$. Here $C^{-1}(S)$ denotes the inverse image of the set $S$ under $C$, defined by $C^{-1}(S)=\{x \in$ $\left.\mathcal{H}_{1}: C x \in S\right\}$.

Suppose that the following assumptions hold

Assumption $1 \mathcal{F}: \mathcal{H}_{2} \rightrightarrows \mathcal{H}_{2}$ is maximal monotone.

Assumption 2 One of the followings holds:

(i) $B$ is bijective;

(ii) $\operatorname{rge}(C) \cap \operatorname{int}(\operatorname{rge}(D+\mathcal{F})) \neq \emptyset$.

Theorem 1 Let Assumptions 1, 2 hold and suppose that $(A-k I, B, C, D)$ is I-passive for some $k \geq 0$. Then for each $x_{0} \in \mathcal{H}_{1}$ such that $x_{0} \in C^{-1}(\operatorname{rge}(D+\mathcal{F}))$, there exists a unique global solution $x\left(\cdot ; x_{0}\right)$ of the Lur'e dynamical system $(\mathcal{S})$. Furthermore, the solutions continuously depend on the initial conditions in the sense that for $x_{0}, x_{1} \in C^{-1}(\operatorname{rge}(D+\mathcal{F}))$, and $t \geq 0$, one has

$$
\left\|x\left(t ; x_{0}\right)-x\left(t ; x_{1}\right)\right\| \leq e^{k t}\left\|x_{0}-x_{1}\right\| .
$$

Proof Fix $T>0$. On $[0, T]$ the system $(\mathcal{S})$ is equivalent to the following differential inclusion

$$
\dot{x}(t) \in k x(t)+A_{1} x(t)-B(D+\mathcal{F})^{-1}(C x(t)) \text { a.e. } t \in[0, T], x(0)=x_{0},
$$

where $A_{1}:=A-k I$. Since $D+\mathcal{F}$ is maximal monotone, then so is $S:=(D+\mathcal{F})^{-1}$. The Yosida approximation of $S$ of index $\mu>0$ is $S_{\mu}=\left(\mu I+S^{-1}\right)^{-1}=(\mu I+D+\mathcal{F})^{-1}$ is Lipschitz continuous. Let us approximate (10) by the following ODE

$$
\dot{x}_{\mu}(t)=k x_{\mu}(t)+A_{1} x_{\mu}(t)-B S_{\mu}\left(C x_{\mu}(t)\right) \quad \forall t \in[0, T], x_{\mu}(0)=x_{0} .
$$

By the Cauchy-Lipschitz Theorem, (11) has a unique differentiable solution $x_{\mu}(\cdot)$. Let $H_{\mu}(x):=-A_{1} x+B S_{\mu}(C x), x \in \mathcal{H}_{1}$. Let us prove that $H_{\mu}$ is monotone. In fact, let $x_{i} \in$ $\mathcal{H}_{1}$ and $y_{i}=S_{\mu}\left(C x_{i}\right)=(\mu I+D+\mathcal{F})^{-1}\left(C x_{i}\right), i=1,2$. Then $C x_{i}-D y_{i} \in(\mathcal{F}+\mu I)\left(y_{i}\right)$. Using the monotonicity of $\mathcal{F}+\mu I$, we get

$$
\left\langle C\left(x_{1}-x_{2}\right)-D\left(y_{1}-y_{2}\right), y_{1}-y_{2}\right\rangle \geq 0 .
$$

Since $\left(A_{1}, B, C, D\right)$ is $I$-passive, we have $\left\langle-A_{1}\left(x_{1}-x_{2}\right), x_{1}-x_{2}\right\rangle+\left\langle B\left(y_{1}-y_{2}\right), x_{1}-x_{2}\right\rangle \geq\left\langle C\left(x_{1}-x_{2}\right)-D\left(y_{1}-y_{2}\right), y_{1}-y_{2}\right\rangle \geq 0$.

Hence $H_{\mu}$ is monotone. For any $\epsilon>0$, one has

$\frac{1}{2} \frac{d}{d t}\left\|x_{\mu}(t+\epsilon)-x_{\mu}(t)\right\|^{2}=\left\langle\dot{x}_{\mu}(t+\epsilon)-\dot{x}_{\mu}(t), x_{\mu}(t+\epsilon)-x_{\mu}(t) \leq k\left\|x_{\mu}(t+\epsilon)-x_{\mu}(t)\right\|^{2}\right.$. 
From Gronwall's inequality, one obtains

$$
\left\|x_{\mu}(t+\epsilon)-x_{\mu}(t)\right\| \leq\left\|x_{\mu}(\epsilon)-x_{\mu}(0)\right\| e^{k t} \leq\left\|x_{\mu}(\epsilon)-x_{\mu}(0)\right\| e^{k T} .
$$

Dividing both side by $\epsilon$ and taking the limit as $\epsilon \rightarrow 0$, one obtains for all $t \in[0, T]$ that

$$
\left\|\dot{x}_{\mu}(t)\right\| \leq\left\|\dot{x}_{\mu}(0)\right\| e^{k T} \leq\left(\left\|A x_{0}\right\|+\|B\|\left\|S^{0}\left(C x_{0}\right)\right\|\right) e^{k T}=: M_{1} .
$$

Hence for all $\mu>0, \dot{x}_{\mu}(\cdot)$ is uniformly bounded by $M_{1}$ and $x_{\mu}(\cdot)$ is uniformly bounded by $M_{2}:=\left\|x_{0}\right\|+M_{1} T$ on $[0, T]$. Therefore, $B S_{\mu}\left(C x_{\mu}(\cdot)\right)$ is uniformly bounded on $[0, T]$. Let us prove that there exists some $\mu_{0}>0$ such that $\left(S_{\mu}\left(C x_{\mu}(\cdot)\right)\right)_{\mu \leq \mu_{0}}$ is uniformly bounded. Note that this conclusion holds if $B$ is bijective since then $B^{-1}$ is bounded due to the known Bounded Inverse Theorem. Suppose on contrary that there exist sequences $y_{n}=S_{\mu_{n}}\left(C x_{\mu_{n}}\left(t_{n}\right)\right), \mu_{n} \rightarrow 0^{+}$and $t_{n} \in[0, T]$ such that $\left\|y_{n}\right\| \rightarrow+\infty$ as $n \rightarrow+\infty$. Let $\gamma \in \operatorname{rge}(C) \cap \operatorname{int}(\operatorname{rge}(D+\mathcal{F}))$. Then there exists some $r>0$ such that $\gamma+r \mathbb{B}_{\mathcal{H}_{1}} \subset \operatorname{rge}(D+\mathcal{F})=\operatorname{dom}(D+\mathcal{F})^{-1}$ and $(D+\mathcal{F})^{-1}$ is bounded on $\gamma+r \mathbb{B}_{\mathcal{H}_{1}}$ by some constant $M_{3}>0$ (Proposition 2). Set $x_{n}=\gamma+r \xi_{n}$, where $\xi_{n}=y_{n} /\left\|y_{n}\right\|$ and $z_{n} \in(D+\mathcal{F})^{-1}\left(x_{n}\right)$. It is clear that the sequence $\left(z_{n}\right)$ is bounded by $M_{3}$. One has $x_{n}-D z_{n} \in \mathcal{F}\left(z_{n}\right)$ and $C x_{\mu_{n}}\left(t_{n}\right)-D y_{n}-\mu_{n} y_{n} \in \mathcal{F}\left(y_{n}\right)$. From the monotonicity of $\mathcal{F}$, we get

$$
\left\langle C x_{\mu_{n}}\left(t_{n}\right)-x_{n}-D\left(y_{n}-z_{n}\right)-\mu_{n} y_{n}, y_{n}-z_{n}\right\rangle \geq 0 \text {. }
$$

Dividing both side of (13) by $\left\|y_{n}\right\|^{2}$ and taking the limit as $n \rightarrow+\infty$, one has $\lim _{n \rightarrow+\infty}\left\langle D \xi_{n}, \xi_{n}\right\rangle=0$ since $\left(x_{\mu_{n}}\left(t_{n}\right)\right),\left(x_{n}\right),\left(z_{n}\right)$ are bounded. Note that

$\lim _{n \rightarrow+\infty} B \xi_{n}=0$ due to the boundedness of $\left(B y_{n}\right)$. Hence $\lim _{n \rightarrow+\infty} C^{T} \xi_{n}=0$ thanks to Lemma 1. From (13) and the monotonicity of $D$, we deduce

$$
\left\langle C x_{\mu_{n}}\left(t_{n}\right)-x_{n}, y_{n}-z_{n}\right\rangle \geq \mu_{n}\left\langle y_{n}, y_{n}-z_{n}\right\rangle \geq \mu_{n}\left\langle z_{n}, y_{n}-z_{n}\right\rangle .
$$

Dividing both sides of (14) by $\left\|y_{n}\right\|$, then taking the liminf and noting that

$\lim _{n \rightarrow+\infty}\left\langle C x_{\mu_{n}}\left(t_{n}\right), \xi_{n}\right\rangle=\lim _{n \rightarrow+\infty}\left\langle x_{\mu_{n}}\left(t_{n}\right), C^{T} \xi_{n}\right\rangle=0$, one obtains:

$$
\liminf _{n \rightarrow+\infty}\left\langle-x_{n}, \xi_{n}\right\rangle \geq 0 .
$$

Thus, $0 \geq \limsup _{n \rightarrow+\infty}\left\langle x_{n}, \xi_{n}\right\rangle=\lim _{n \rightarrow+\infty}\left\langle\gamma+r \xi_{n}, \xi_{n}\right\rangle=r$ since $\gamma \in \operatorname{rge}(C)$ and $\lim _{n \rightarrow+\infty} C^{T} \xi_{n}=$ 0 , which is a contradiction. Hence $\left(S_{\mu}\left(C x_{\mu}(\cdot)\right)\right)_{\mu \leq \mu_{0}}$ is uniformly bounded by some constant $M_{4}>0$.

Next, we will prove that $\left(x_{\mu}(\cdot)\right)_{\mu \leq \mu_{0}}$ is a Cauchy sequence in $C\left([0, T] ; \mathcal{H}_{1}\right)$. Indeed, let $0<\mu, v \leq \mu_{0}$, we have

$$
\begin{aligned}
& \frac{1}{2} \frac{d}{d t}\left\|x_{v}(t)-x_{\mu}(t)\right\|^{2} \\
= & \left\langle\dot{x}_{v}(t)-\dot{x}_{\mu}(t), x_{v}(t)-x_{\mu}(t)\right\rangle \\
= & \left\langle A\left(x_{v}(t)-x_{\mu}(t)\right)-B\left(y_{v}(t)-y_{\mu}(t)\right), x_{v}(t)-x_{\mu}(t)\right\rangle \\
\leq & k\left\|x_{v}(t)-x_{\mu}(t)\right\|^{2}+\left\langle D\left(y_{v}(t)-y_{\mu}(t)\right)-C\left(x_{v}(t)-x_{\mu}(t)\right), y_{v}(t)-y_{\mu}(t)\right\rangle,
\end{aligned}
$$

where $y_{\nu}(t)=S_{\nu}\left(C x_{\lambda}(t)\right), y_{\mu}(t)=S_{\mu}\left(C x_{\mu}(t)\right)$ and the last inequality comes from the passivity of $\left(A_{1}, B, C, D\right)$. On the other hand, one has

$$
C x_{v}(t)-D y_{v}(t)-v y_{v}(t) \in \mathcal{F}\left(y_{v}(t)\right) \text { and } C x_{\mu}(t)-D y_{\mu}(t)-\mu y_{\mu}(t) \in \mathcal{F}\left(y_{\mu}(t)\right) \text {. }
$$

Using the monotonicity of $\mathcal{F}$, we get

$$
\left\langle D\left(y_{\nu}(t)-y_{\mu}(t)\right)-C\left(x_{v}(t)-x_{\mu}(t)\right), y_{\nu}(t)-y_{\mu}(t)\right\rangle \leq\left\langle-v y_{v}(t)+\mu y_{\mu}(t), y_{\nu}(t)-y_{\mu}(t)\right\rangle \text {. }
$$


Therefore,

$$
\begin{aligned}
& \frac{1}{2} \frac{d}{d t}\left\|x_{v}(t)-x_{\mu}(t)\right\|^{2} \\
\leq & k\left\|x_{v}(t)-x_{\mu}(t)\right\|^{2}+\left\langle D\left(y_{v}(t)-y_{\mu}(t)\right)-C\left(x_{v}(t)-x_{\mu}(t)\right), y_{v}(t)-y_{\mu}(t)\right\rangle \\
\leq & k\left\|x_{v}(t)-x_{\mu}(t)\right\|^{2}+\left\langle-v y_{v}(t)+\mu y_{\mu}(t), y_{v}(t)-y_{\mu}(t)\right\rangle \\
= & k\left\|x_{v}(t)-x_{\mu}(t)\right\|^{2}+\left\langle(\mu-v) y_{\mu}(t), y_{v}(t)-y_{\mu}(t)\right\rangle-v\left\|y_{\lambda}(t)-y_{\mu}(t)\right\|^{2} \\
\leq & k\left\|x_{v}(t)-x_{\mu}(t)\right\|^{2}+|v-\mu|\left\|y_{\mu}(t)\right\|\left\|y_{v}(t)-y_{\mu}(t)\right\| \\
\leq & k\left\|x_{v}(t)-x_{\mu}(t)\right\|^{2}+2 M_{4}^{2}|v-\mu| .
\end{aligned}
$$

Using Gronwall's inequality and noting that $x_{\lambda}(0)=x_{\mu}(0)=x_{0}$, one has

$$
\left\|x_{v}(t)-x_{\mu}(t)\right\|^{2} \leq 4 M_{4}^{2}|v-\mu| \int_{0}^{t} e^{2 k(t-s)} d s \leq 4 M_{4}^{2} T|v-\mu| e^{2 k T}, \forall t \in[0, T]
$$

or equivalently

$$
\left\|x_{v}(t)-x_{\mu}(t)\right\| \leq 2 M_{4} e^{k T} \sqrt{|v-\mu| T}, \forall t \in[0, T] .
$$

As a consequence, $\left(x_{\mu}(\cdot)\right)_{\mu \leq \mu_{0}}$ is a Cauchy sequence in $C\left([0, T] ; \mathcal{H}_{1}\right)$ and there exists a (Lipschitz continuous) function $x(\cdot) \in C\left([0, T] ; \mathcal{H}_{1}\right)$ such that $x_{\mu}(\cdot)$ converges to $x(\cdot)$ in $C\left([0, T] ; \mathcal{H}_{1}\right)$. Let $v \rightarrow 0$ in $(16)$, one has

$$
\left\|x_{\mu}(t)-x(t)\right\| \leq 2 M_{4} e^{k T} \sqrt{\mu T}, \quad \forall t \in[0, T] .
$$

For each $t \in[0, T],\left(S_{\mu}\left(C x_{\mu}(t)\right)\right)_{\mu \leq \mu_{0}}$ is bounded and $C x_{\mu}(t) \rightarrow C x(t)$ hence $C x(t) \in$ $\operatorname{dom} S=\operatorname{rge}(D+\mathcal{F})$ (Proposition 1$)$. Since $\left(\dot{x}_{\mu}(\cdot)\right)$ is uniformly bounded by $M_{1}$ on $[0, T]$, there exists a subsequence, still denoted by $\left(\dot{x}_{\mu}(\cdot)\right)$, such that $\left(\dot{x}_{\mu}(\cdot)\right)$ converges weakly to $\dot{x}(\cdot)$ in $L^{2}\left([0, T], \mathcal{H}_{1}\right)$ (see e.g. Theorem 4, p. 23 [15]). In addition, $\left(S_{\mu}\left(C x_{\mu}(\cdot)\right)\right)_{\mu \leq \mu_{0}}$ is uniformly bounded by $M_{4}$, there exists some $\gamma(\cdot) \in L^{2}\left([0, T], \mathcal{H}_{1}\right)$ and a subsequence, still denoted by itself, such that $\left(S_{\mu}\left(C x_{\mu}(\cdot)\right)\right)_{\mu \leq \mu_{0}}$ converges weakly to $\gamma(\cdot)$ in $L^{2}\left([0, T], \mathcal{H}_{1}\right)$. Note that

$$
\begin{aligned}
\left\|J_{\mu}^{S}\left(C x_{\mu}(t)\right)-C x(t)\right\| & \leq\left\|J_{\mu}^{S}\left(C x_{\mu}(t)\right)-C x_{\mu}(t)\right\|+\left\|C x_{\mu}(t)-C x(t)\right\| \\
& \leq \mu\left\|S_{\mu}\left(C x_{\mu}(t)\right)\right\|+\|C\|\left\|x_{\mu}(t)-x(t)\right\| \\
& \leq \mu M_{4}+2 M_{4}\|C\| e^{k T} \sqrt{\mu T} .
\end{aligned}
$$

Hence $J_{\mu}^{S}\left(C x_{\mu}(\cdot)\right)_{\mu \leq \mu_{0}}$ converges strongly to $C x(\cdot)$ in $C\left([0, T], \mathcal{H}_{1}\right)$, thus in $L^{2}\left([0, T], \mathcal{H}_{1}\right)$ also. Furthermore, one has the relation

$$
S_{\mu}\left(C x_{\mu}(t)\right) \in S\left(J_{\mu}^{S}\left(C x_{\mu}(t)\right)\right), \quad \forall t \in[0, T],
$$

which implies that (Proposition 2)

$$
\gamma(t) \in S(C x(t))=(D+\mathcal{F})^{-1}(C x(t)) \text { for almost all } t \in[0, T] .
$$

On the other hand, from (11), one deduce that

$$
\dot{x}(t)=A x(t)-B \gamma(t) \text { a.e. } t \in[0, T] .
$$

It means that $x(\cdot)$ is a solution of (10). Let $H(x):=-A_{1} x+B S(C x), x \in \mathcal{H}_{1}$, then the monotonicity of $H$ can be proved similarly for $H_{\mu}$. Thus (10) has at most a solution. Therefore, $x(\cdot)$ is the unique solution of $(10)$, or equivalently of $(\mathcal{S})$, on $[0, T]$. Since $T$ is arbitrary, the conclusion follows which completes the global existence and uniqueness of solutions. 
Let $x_{0}, x_{1} \in C^{-1}(\operatorname{rge}(D+\mathcal{F}))$. From the monotonicity of $H(x)$, one has

$\frac{1}{2} \frac{d}{d t}\left\|x\left(t ; x_{0}\right)-x\left(t ; x_{1}\right)\right\|^{2}=\left\langle\dot{x}\left(t ; x_{0}\right)-\dot{x}\left(t ; x_{1}\right), x\left(t ; x_{0}\right)-x\left(t ; x_{1}\right) \leq k\left\|x\left(t ; x_{0}\right)-x\left(t ; x_{1}\right)\right\|^{2}\right.$.

Using Gronwall's inequality, for all $t \geq 0$, one has

$$
\left\|x\left(t ; x_{0}\right)-x\left(t ; x_{1}\right)\right\| \leq\left\|x_{0}-x_{1}\right\| e^{k t},
$$

and the proof of Theorem 1 is thereby completed.

Remark 2 i) In the case of finite dimension, the interior in Assumption 2 can be reduced to relative interior.

ii) If there exists some symmetric positive definite $P \in \mathcal{L}(\mathcal{H})$ such that $P B=C^{T}$, then it is easy to see that $(A-k I, B, C, D)$ is $P$-passive for $k$ large enough. However, in this case, from the proof of Theorem 1, Assumption 2 (ii) and the maximality of $\mathcal{F}+D$ are sufficient for the well-posedness without requiring the monotonicity of $\mathcal{F}$ or $D$.

iii) In [7], the existence and uniqueness of solutions are acquired by proving the operator $H: \mathcal{H}_{1} \rightrightarrows \mathcal{H}_{1}, x \mapsto H(x)=-A_{1} x+B S(C x)$, where $A_{1}=A-k I$, is maximal monotone. The authors use a characterization of maximal monotonicity which cannot be extended for infinite-dimensional spaces. Our approach is based on a regularization procedure; however it also allows to obtain the maximal monotonicity of $H$, even in reflexive Banach spaces.

(iv) If $D$ is positive definite then the proof is much easier since then $(\mathcal{F}+D)^{-1}$ is single-valued Lipschitz continuous. Hence $-A+B(\mathcal{F}+D)^{-1}(C \cdot)$ is single-valued monotone, Lipschitz continuous and thus is maximal monotone. Therefore, the case when $D$ is monotone but not positive definite is more interesting.

(v) The operator $A$ does not play an important role in Theorem 1. Indeed, the result is still true if $A$ is replaced by any Lipschitz continuous function satisfying the passivity condition (3). In contrast, the role of the operators $B, C, D$ can be seen in Assumption 2. It is easy to realize that Assumption $2(i)$ and $(i i)$ are two kind of independently sufficient conditions, which do not imply to each other. For example in $\mathbb{R}^{2}$, let us take

$$
-A=B=I_{2}=\left(\begin{array}{ll}
1 & 0 \\
0 & 1
\end{array}\right), C=\left(\begin{array}{ll}
1 & 0 \\
0 & 0
\end{array}\right), D=\mathcal{F}=\left(\begin{array}{ll}
0 & 0 \\
0 & 1
\end{array}\right),
$$

then $B$ is bijective but $\operatorname{int}(\operatorname{rge}(D+\mathcal{F}))=\emptyset$. On the other hand, if we take

$$
-A=I_{2}, C=0_{2}, B=D=\left(\begin{array}{ll}
0 & 0 \\
0 & 1
\end{array}\right), \mathcal{F}=\left(\begin{array}{ll}
1 & 0 \\
0 & 0
\end{array}\right),
$$

then $\operatorname{rge}(C) \cap \operatorname{int}(\operatorname{rge}(D+\mathcal{F})) \neq \emptyset$ but $B$ is not bijective.

Corollary 1 Let Assumptions 1, 2 hold and suppose that $(A-k I, B, C, D)$ is P-passive for some $k \geq 0$ and symmetric positive definite $P \in \mathcal{L}\left(\mathcal{H}_{1}\right)$. Then for each $x_{0} \in \mathcal{H}_{1}$ such that $x_{0} \in C^{-1}(\operatorname{rge}(D+\mathcal{F}))$, there exists a unique solution $x\left(\cdot ; x_{0}\right)$ of the Lur'e dynamical system $(\mathcal{S})$. In addition, the solutions continuously depend on the initial conditions.

Proof Using classical technique of changing variable, one can reduce the $P$-passivity into $I$-passivity. Indeed, let $R$ be the square root operator of $P$, then $R \in \mathcal{L}\left(\mathcal{H}_{1}\right)$ is also symmet- 
ric and positive definite. The system $(\mathcal{S})$ can be recast in the following form of first order differential inclusion

$$
\dot{x}(t) \in A x(t)-B(D+\mathcal{F})^{-1}(C x(t)) \text { a.e. } t \geq 0,
$$

which is equivalent to

$$
R \dot{x} \in k R x+R A_{1} R^{-1} R x-R B(D+\mathcal{F})^{-1}\left(C R^{-1} R x\right),
$$

where $A_{1}=A-k I$ and $\left(A_{1}, B, C, D\right)$ is $P$-passive. Setting $z=R x$, we have

$$
\dot{z} \in k z+R A_{1} R^{-1} z-R B(D+\mathcal{F})^{-1}\left(C R^{-1} z\right) .
$$

By setting $(\bar{A}, \bar{B}, \bar{C})=\left(R A_{1} R^{-1}, R B, C R^{-1}\right)$, we derive

$$
\dot{z} \in k z+\bar{A} z-\bar{B}(D+\mathcal{F})^{-1}(\bar{C} z) .
$$

In addition, for all $y_{1} \in \mathcal{H}_{1}, y_{2} \in \mathcal{H}_{2}$, we have

$$
\begin{aligned}
& \left\langle\bar{A} y_{1}, y_{1}\right\rangle+\left\langle\left(\bar{B}-\bar{C}^{T}\right) y_{2}, y_{1}\right\rangle-\left\langle D y_{2}, y_{2}\right\rangle \\
= & \left\langle P A_{1} \bar{y}_{1}, \bar{y}_{1}\right\rangle+\left\langle\left(P B-C^{T}\right) y_{2}, \bar{y}_{1}\right\rangle-\left\langle D y_{2}, y_{2}\right\rangle \leq 0,
\end{aligned}
$$

with $\bar{y}_{1}=R^{-1} y_{1}$. We conclude that $(\bar{A}, \bar{B}, \bar{C}, D)$ is $I$-passive. The desired result follows then by Theorem 1 .

\section{Stability Analysis}

In this section, we investigate the stability of equilibria and extend the Krasovskii-LaSalle invariance principle for our system $(\mathcal{S})$. Let $\mathcal{W}$ be the set of equilibria of $(\mathcal{S})$, defined by

$$
\mathcal{W}=\left\{\bar{x} \in \mathcal{A} \text { and } 0 \in A \bar{x}-B(D+\mathcal{F})^{-1}(C \bar{x})\right\},
$$

where $\mathcal{A}=\left\{x \in \mathcal{H}_{1}: x \in C^{-1}(\operatorname{rge}(D+\mathcal{F}))\right\}$. Assume that the well-posedness of the system is already acquired and for a given initial point $x_{0} \in \mathcal{H}$, let $x\left(\cdot ; x_{0}\right)$ be the unique solution of $(\mathcal{S})$. First, we assume the following assumption related to the generalized sector nonlinearities, a familiar term in stability analysis of Lur'e dynamical systems [3].

Assumption $30 \in \mathcal{F}(0)$.

Remark 3 i) If Assumption 3 holds, then the monotonicity of $\mathcal{F}$ implies that $\mathcal{F}$ is in the sector $[0,+\infty]$, i.e for all $y \in \mathcal{H}_{2}, y^{*} \in \mathcal{F}(y)$, we have $\left\langle y, y^{*}\right\rangle \geq 0$.

ii) Assumption 3 also ensures that $0 \in \mathcal{W}$ and $x(t ; 0)=0$ for all $t \geq 0$.

Let us recall some basic definitions of Lyapunov stability theory [4, 19] adapted to system $(\mathcal{S})$.

Definition 4 The equilibrium point $x=0$ is said to be stable if

$$
\forall \varepsilon>0, \exists \delta>0 \text { such that for all } x_{0} \in \mathbb{B}_{\delta} \cap \mathcal{A} \text {, then }\left\|x\left(t ; x_{0}\right)\right\| \leq \varepsilon, \forall t \geq 0 .
$$

Definition 5 The equilibrium point $x=0$ is said to be attractive if

$$
\forall \varepsilon>0, \exists \delta>0 \text { such that for all } x_{0} \in \mathbb{B}_{\delta} \cap \mathcal{A} \text {, then } \lim _{t \rightarrow \infty}\left\|x\left(t ; x_{0}\right)\right\|=0 .
$$

If this is true for all $x_{0} \in \mathcal{A}$ then $x=0$ is said globally attractive. 
Definition 6 If the trivial equilibrium point is stable and attractive, it is called asymptotic stable; if it is stable and globally attractive, it is called globally asymptotically stable.

Definition 7 The equilibrium point $x=0$ is said to be exponentially stable if

$$
\exists \delta>0, \varepsilon>0, a \geq 0 \text { such that for all } x_{0} \in \mathbb{B}_{\delta} \cap \mathcal{A} \text {, then }\left\|x\left(t ; x_{0}\right)\right\| \leq a e^{-\varepsilon t} .
$$

If this is true for all $x_{0} \in \mathcal{A}$ then $x=0$ is said globally exponentially stable.

Definition 8 The equilibrium point $x=0$ is said to be finite-time stable if it is stable and for all $x_{0} \in \mathcal{A}$, there exists $t_{f}<+\infty$ such that

$$
x\left(t ; x_{0}\right)=0 \text { for all } t \geq t_{f} .
$$

Theorem 2 Let Assumption 3 hold and suppose that $(A, B, C, D)$ is $P$-passive. Then the origin is stable.

Proof Define the Lyapunov function $V(x)=\frac{1}{2}\langle P x, x\rangle$ which is positive definite. It is sufficient to prove that the derivative of $V(\cdot)$ along the trajectories of system $(\mathcal{S})$ is nonpositive, i.e., $V(\cdot)$ is non-increasing along the system trajectories. Indeed, we have

$$
\begin{aligned}
\dot{V}(x) & =\left\langle V^{\prime}(x), \dot{x}\right\rangle=\langle P \dot{x}, x\rangle=\langle P A x, x\rangle+\langle P B \lambda, x\rangle \\
& \leq\langle C x, \lambda\rangle+\langle D \lambda, \lambda\rangle=\langle C x+D \lambda, \lambda\rangle \leq 0,
\end{aligned}
$$

where $\lambda \in-(D+\mathcal{F})^{-1}(C x)$, the first inequality comes from the passivity of $(A, B, C, D)$ and the second inequality is deduced from the fact that $C x+D \lambda \in \mathcal{F}(-\lambda)$ and $\mathcal{F}$ is in the sector $[0,+\infty]$. Given $x_{0} \in \mathcal{A}$, let $x(\cdot):=x\left(\cdot ; x_{0}\right)$ then $V(x(t)) \leq V\left(x_{0}\right) \leq \frac{1}{2}\|P\| \cdot\left\|x_{0}\right\|^{2}$, where $\|P\|$ is the operator norm of $P$. Note that $V(x) \geq \frac{1}{2} \lambda_{\min }(P)\|x\|^{2}$ where $\lambda_{\min }(P)>0$ is the smallest eigenvalue of $P$. Hence for all $t \geq 0$

$$
\|x(t)\| \leq \sqrt{\frac{\|P\|}{\lambda_{\min }(P)}}\left\|x_{0}\right\|,
$$

which implies the stability of the origin.

Remark 4 The Lyapunov function $V(\cdot)$ defined above is differentiable. In [20], the authors give a general sufficient condition in Hilbert space under which two extended proper lower semi-continuous functions form a Lyapunov pair.

Theorem 3 Let Assumption 3 hold and suppose that $(A, B, C, D)$ is strictly P-passive. Then the origin is globally exponentially stable.

Proof Let $\varepsilon>0$ be such that $(A+\varepsilon I, B, C, D)$ is $P$-passive. Define the same Lyapunov function $V(\cdot)$ as in Theorem 2. Then the derivative of $V(\cdot)$ along the trajectories of the system is

$$
\begin{aligned}
\dot{V}(x) & =\langle P A x, x\rangle+\langle P B \lambda, x\rangle=\langle P(A+\varepsilon I) x, x\rangle+\langle P B \lambda, x\rangle-\varepsilon x^{T} P x \\
& \leq\langle C x+D \lambda, \lambda\rangle-2 \varepsilon V(x) \leq-2 \varepsilon V(x),
\end{aligned}
$$


where $\lambda \in-(D+\mathcal{F})^{-1}(C x)$ and the first inequality comes from the passivity of $(A+$ $\varepsilon I, B, C, D)$. Given $x_{0} \in \mathcal{A}$ and let $x(\cdot):=x\left(\cdot ; x_{0}\right)$ be the unique solution of $(\mathcal{S})$. Then we obtain

$$
\frac{d}{d t} V(x(t)) \leq-2 \varepsilon V(x(t)) \text { for a.e. } t \geq 0 .
$$

Using a simple integration and note that $V(x) \geq \frac{1}{2} \lambda_{\min }(P)\|x\|^{2}$, one infers

$$
\frac{1}{2} \lambda_{\min }(P)\|x(t)\|^{2} \leq V(x(t)) \leq V\left(x_{0}\right) e^{-2 \varepsilon t},
$$

which implies that

$$
\|x(t)\| \leq \sqrt{\frac{2 V\left(x_{0}\right)}{\lambda_{\min }(P)}} e^{-\varepsilon t} .
$$

Hence the origin is globally exponentially stable.

The following theorem provides an asymptotical result with weaker assumption than strict passivity. In contrast, the rate of convergence cannot be estimated.

Theorem 4 Suppose for any $0<\beta \leq \alpha$, there exists $\varepsilon>0$ such that

$$
\sup _{\beta \leq\|x\| \leq \alpha, y \in-(D+\mathcal{F})^{-1}(C x)}\{\langle P A x, x\rangle+\langle P B y, x\rangle\} \leq-\varepsilon .
$$

Then the origin is globally asymptotically stable.

Proof From (27), it is easy to obtain that

$$
\sup _{x \in \mathcal{H}, y \in-(D+\mathcal{F})^{-1}(C x)}\{\langle P A x, x\rangle+\langle P B y, x\rangle\} \leq 0 .
$$

Then the derivative of $V(\cdot)$ along the trajectories of system $(\mathcal{S})$ is non-positive since

$$
\dot{V}(x)=\langle P A x, x\rangle+\langle P B \lambda, x\rangle,
$$

where $\lambda \in-(D+\mathcal{F})^{-1}(C x)$. Hence, the origin is stable. Given $x_{0} \in \mathcal{A}$ and let $x(\cdot):=$ $x\left(\cdot ; x_{0}\right)$ be the unique solution of $(\mathcal{S})$. Let $c=V\left(x_{0}\right)$ and define $\Omega_{c}=\{x \in \mathcal{H}: V(x) \leq c\}$. Since $V(\cdot)$ is non-increasing along the system trajectories, one infers that $x(t) \in \Omega_{c}$ for all $t \geq 0$. It is easy to see that $\Omega_{c} \subset \mathbb{B}_{\alpha}$ where $\alpha=\sqrt{\frac{2 c}{\lambda_{\min }(P)}}$. Note that $V(x(\cdot))$ is nonincreasing and bounded from below by 0 . Hence there exists a non-negative real number $b$ such that

$$
V(x(t)) \rightarrow b \text { as } t \rightarrow+\infty .
$$

It is sufficient to prove that $b=0$ since $V(x) \geq \frac{1}{2} \lambda_{\min }(P)\|x\|^{2}$. Arguing by contradiction, suppose that $b>0$. Then we can find $\beta>0$ such that $\mathbb{B}_{\beta} \subset \Omega_{b}$. Hence for all $t \geq$ $0,\|x(t)\| \geq \beta$. Then from (27), there exists $\varepsilon>0$ such that for a.e. $t \geq 0$,

$$
\frac{d}{d t} V(x(t))=\langle P A x(t), x(t)\rangle+\langle P B \lambda(t), x(t)\rangle \leq-\varepsilon,
$$

where $\lambda(t) \in-(D+\mathcal{F})^{-1}(C x(t))$. Therefore for all $t \geq 0$

$$
V(x(t))=V\left(x_{0}\right)+\int_{0}^{t} \frac{d}{d s} V(x(s)) \leq V\left(x_{0}\right)-\varepsilon t,
$$

which is a contradiction and the proof is completed. 
Theorem 5 Suppose that there exists $\varepsilon>0$ such that for all $x \in \mathcal{H}_{1}$ :

$$
\sup _{y \in-(D+\mathcal{F})^{-1}(C x)}\{\langle P A x, x\rangle+\langle P B y, x\rangle\} \leq-\varepsilon\|x\| .
$$

Then the origin is finite-time stable.

Proof It is not difficult to see that condition (29) implies condition (27). Hence the origin is globally asymptotically stable, i.e. for each $x_{0} \in \mathcal{A}$, we have

$$
\lim _{t \rightarrow \infty}\left\|x\left(t ; x_{0}\right)\right\|=0 .
$$

The derivative of $V(\cdot)$ along the trajectories of system $(\mathcal{S})$ is

$$
\dot{V}(x)=\langle P A x, x\rangle+\langle P B \lambda, x\rangle,
$$

where $\lambda \in-(D+\mathcal{F})^{-1}(C x)$. Thus, from (29), one infers

$$
\dot{V}(x) \leq-\varepsilon\|x\| \text {. }
$$

Note that $V(x) \leq \frac{1}{2}\|P\| \cdot\|x\|^{2}$, where $\|P\|$ is the norm of $P$. Consequently

$$
\dot{V}(x) \leq-k V^{1 / 2}(x),
$$

where $k=\varepsilon \sqrt{2 /\|P\|}$. It means that for almost all $t \geq 0$, one has

$$
\frac{d}{d t} V\left(x\left(t ; x_{0}\right)\right)+k V^{1 / 2}\left(x\left(t ; x_{0}\right)\right) \leq 0 .
$$

Assume that for all $t \geq 0, w(t):=V\left(x\left(t ; x_{0}\right)\right)>0$. Dividing (30) by $2 \sqrt{w(t)}$ and integrating from 0 to $t$, we have for all $t \geq 0$,

$$
w(t)-w(0) \leq-k t / 2,
$$

which is a contradiction. Therefore, there exists $t_{f}<+\infty$ such that $w\left(t_{f}\right)=0$. Since $w(\cdot)$ is non-negative and non-increasing, we deduce that $w(t)=0$ for all $t \geq t_{f}$. Then it is easy to conclude that $x\left(t ; x_{0}\right)=0$ for all $t \geq t_{f}$. The result has been proved.

Remark 5 We give some cases such that condition (29) of Theorem 5 holds. For example, consider $P=I, B=C=\sqrt{\varepsilon} I, D=0, A$ is negative semidefinite and $\mathcal{F}=N_{\mathbb{B}_{\mathcal{H}_{2}}}$ the normal cone of the unit ball. Then it is easy to compute that

$$
\mathcal{F}^{-1}(x)=\partial\|x\|=\operatorname{Sign}(x)= \begin{cases}x /\|x\| & \text { if } x \neq 0, \\ \mathbb{B}_{\mathcal{H}_{2}} & \text { if } x=0 .\end{cases}
$$

Clearly (29) holds for $x=0$. If $x \neq 0$ then

$$
\sup _{y \in-(D+\mathcal{F})^{-1}(C x)}\{\langle P A x, x\rangle+\langle P B y, x\rangle\} \leq\left\langle-\frac{\varepsilon x}{\|x\|}, x\right\rangle=-\varepsilon\|x\| .
$$

In some cases, the system $(A, B, C, D)$ is passive but neither strictly passive nor satisfy (27). Thus it is natural to extend the Krasovskii-LaSalle invariance principle to obtain the asymptotical property of the system. From now, let us consider the system $(\mathcal{S})$ in finitedimensional spaces $\left(\mathcal{H}_{1}=\mathbb{R}^{n}\right.$ and $\left.\mathcal{H}_{2}=\mathbb{R}^{m}\right)$. Let $x_{0} \in \mathcal{A}$ and $x\left(\cdot ; x_{0}\right)$ be the solution of $(\mathcal{S})$, denote the orbit by

$$
\gamma\left(x_{0}\right):=\left\{x\left(t ; x_{0}\right): t \geq 0\right\} \subset \mathcal{A},
$$


and the limit set by

$$
\Lambda\left(x_{0}\right):=\left\{p \in \mathbb{R}^{n}: \exists\left\{t_{i}\right\}, t_{i} \rightarrow \infty \text { as } i \rightarrow \infty \text { and } x\left(t_{i} ; x_{0}\right) \rightarrow p\right\} .
$$

A set $S \subset \mathcal{A}$ is said invariant if and only if for all $x_{0} \in S$, the solution $x\left(\cdot ; x_{0}\right)$ of $(\mathcal{S})$ starting at $x_{0}$ remains in $S$. The following theorem ensures the Lipschitz dependence of the solutions with respect to the initial conditions.

Theorem 6 Let Assumption 3 hold and suppose that $(A, B, C, D)$ is $P$-passive. Then the solutions of $(\mathcal{S})$ are Lipschitz dependent on the initial conditions, i.e., the mapping $x_{0} \mapsto$ $x\left(\cdot ; x_{0}\right)$ is Lipschitz continuous.

Proof Let $x_{0 i} \in \mathcal{A}$ and $x_{i}(\cdot):=x_{i}\left(\cdot ; x_{0 i}\right)$ be the unique solution of $(\mathcal{S})$ satisfying initial condition $x_{i}(0)=x_{0 i}(i=1,2)$. Similarly to the proof of Theorem 2, for almost all $t \geq 0$, one obtains

$$
\begin{aligned}
& \frac{d}{d t}\left\langle\frac{1}{2} P\left(x_{1}(t)-x_{2}(t)\right), x_{1}(t)-x_{2}(t)\right\rangle=\left\langle P\left(\dot{x}_{1}(t)-\dot{x}_{2}(t)\right), x_{1}(t)-x_{2}(t)\right\rangle \\
= & \left\langle P A\left(x_{1}(t)-x_{2}(t)\right), x_{1}(t)-x_{2}(t)\right\rangle+\left\langle P B\left(\lambda_{1}(t)-\lambda_{2}(t)\right), x_{1}(t)-x_{2}(t)\right\rangle \\
\leq & \left\langle C\left(x_{1}(t)-x_{2}(t)\right)+D\left(\lambda_{1}(t)-\lambda_{2}(t)\right), \lambda_{1}(t)-\lambda_{2}(t)\right\rangle \leq 0,
\end{aligned}
$$

where the first inequality comes from the passivity of $(A, B, C, D)$ and the second inequality is deduced from the fact that $C x_{i}(t)+D \lambda_{i}(t) \in \mathcal{F}\left(-\lambda_{i}(t)\right)(i=1,2)$ and $\mathcal{F}$ is monotone. Thus for all $t \geq 0$, we have

$$
\begin{aligned}
& \lambda_{\min }(P)\left\|x_{1}(t)-x_{2}(t)\right\|^{2} \leq\left\langle P\left(x_{1}(t)-x_{2}(t)\right), x_{1}(t)-x_{2}(t)\right\rangle \\
& \leq\left\langle P\left(x_{01}-x_{02}\right), x_{01}-x_{02}\right\rangle \leq\|P\| \cdot\left\|x_{01}-x_{02}\right\|^{2} .
\end{aligned}
$$

Therefore,

$$
\sup _{t \geq 0}\left\|x_{1}(t)-x_{2}(t)\right\| \leq \sqrt{\frac{\|P\|}{\lambda_{\min }(P)}}\left\|x_{01}-x_{02}\right\|,
$$

and the result follows.

Remark 6 Let $x_{0} \in \mathcal{A}$. Then the following results are obtained by using the similar arguments as in $[4,6,18,19]$.

(i) If $\gamma\left(x_{0}\right)$ is bounded, then $\Lambda\left(x_{0}\right) \neq \emptyset$ and $\lim _{t \rightarrow \infty} d\left(x\left(t ; x_{0}\right), \Lambda\left(x_{0}\right)\right)=0$.

(ii) The set of stationary solutions $\mathcal{W}$ is invariant.

(iii) The limit set $\Lambda\left(x_{0}\right)$ is invariant.

(iv) There exists a constant $k \in \mathbb{R}$ such that $V(x)=k$ for all $x \in \Lambda\left(x_{0}\right)$, where $V$ is the Lyapunov function defined in Theorem 2.

Theorem 7 Let Assumption 3 hold. Furthermore, assume that

$$
y \in \mathbb{R}^{m}, y^{*} \in \mathcal{F}(y):\left\langle y^{*}, y\right\rangle=0 \Rightarrow y=0 .
$$

Then for each $x_{0} \in \mathcal{A}$, we have

$$
\lim _{t \rightarrow+\infty} d\left(x\left(t ; x_{0}\right), \mathcal{M}\right)=0,
$$

where $\mathcal{M}$ is the largest invariant subset of

$$
\mathcal{P}=\{x \in \mathcal{A}:\langle P A x, x\rangle=0 \text { and } C x \in \mathcal{F}(0)\} .
$$


Proof Theorem 6 implies that $\gamma\left(x_{0}\right)$ is bounded and hence

$$
\lim _{t \rightarrow \infty} d\left(x\left(t ; x_{0}\right), \Lambda\left(x_{0}\right)\right)=0 .
$$

Since $\Lambda\left(x_{0}\right)$ is invariant, it is sufficient to prove that $\Lambda\left(x_{0}\right) \subset \mathcal{P}$ (Remark 6-iii). Let $z \in$ $\Lambda\left(x_{0}\right)$, then there exists a real constant $k$ such that (Remark 6-iv):

$$
V(x(t ; z))=k \text { for all } t \geq 0 .
$$

Therefore for almost all $t \geq 0$,

$$
\begin{aligned}
0 & =\frac{d}{d t} V(x(t ; z))=\langle P \dot{x}(t ; z), x(t ; z)\rangle=\langle P A x(t ; z), x(t ; z)\rangle+\langle P B \lambda(t), x(t ; z)\rangle \\
& \leq\langle C x(t ; z)+D \lambda(t), \lambda(t)\rangle \leq 0,
\end{aligned}
$$

where $\lambda(t) \in-(D+\mathcal{F})^{-1}(C x(t))$.

Consequently,

$$
\langle C x(t ; z)+D \lambda(t), \lambda(t)\rangle=0 .
$$

Using (32), one has $\lambda(t)=0$ for almost all $t \geq 0$.

Hence,

$$
\langle P A x(t ; z), x(t ; z)\rangle=0 \text { and } C x(t ; z) \in \mathcal{F}(0) \text { for almost all } t \geq 0 .
$$

Since $x(\cdot ; z)$ is continuous and the graph of $\mathcal{F}$ is closed, by letting $t \rightarrow+\infty$, we have $z=x(0 ; z) \in \mathcal{P}$, which completes the proof of Theorem 7 .

Remark 7 i) The condition (32) holds for a large class of maximal monotone operators. In particular it holds for the set-valued function $\operatorname{Sign}(\cdot)$ in $\mathbb{R}^{m}$, an important function in control theory. Theorem 7 is an extension of Theorem 5 in [6] when $\mathcal{F}=\partial \Phi^{*,-}$ for some proper, convex lower semi-continuous function $\Phi^{*,-}$. It is easy to see that the condition (32) in Theorem 7 is then weaker than the condition in Theorem 5 [6]. Indeed, let some $y \in R^{m}, y^{*} \in \mathcal{F}(y)=\partial \Phi^{*,-}(y)$ such that $\left\langle y^{*}, y\right\rangle=0$. If the condition in Theorem 5 [6] holds, then $0=\left\langle y^{*}, y-0\right\rangle \geq \Phi^{*,-}(y)-\Phi^{*,-}(0) \geq 0$ and it implies that $y=0$. Thus the condition (32) in Theorem 7 is satisfied. Furthermore, one can see that the largest invariant subset here is also improved.

ii) The passivity of $(A, B, C, D)$ implies that $P A$ is negative semidefinite, see Lemma 1. It is easy to see that if $P A$ is negative definite, then the origin is globally asymptotically stable since $\mathcal{M}=\{0\}$.

iii) If the assumptions of Theorem 7 hold, then the set of stationary solutions $\mathcal{W}=$ $\operatorname{ker}(A) \cap C^{-1}(\mathcal{F}(0)) \subset \mathcal{P}$. Indeed let $x_{0} \in \mathcal{W}$, then there exists $\lambda \in \mathcal{H}_{2}$ such that $A x_{0}=-B \lambda$ and $-\lambda \in(\mathcal{F}+D)^{-1}\left(C x_{0}\right)$, or equivalently $C x_{0}+D \lambda \in \mathcal{F}(-\lambda)$. Hence,

$$
0=\left\langle P A x_{0}, x_{0}\right\rangle+\left\langle P B \lambda, x_{0}\right\rangle \leq\left\langle C x_{0}+D \lambda, \lambda\right\rangle \leq 0,
$$

which implies that $\lambda=0$ and thus $A x_{0}=0, C x_{0} \in \mathcal{F}(0)$. Therefore $\mathcal{W} \subset$ $\operatorname{ker}(A) \cap C^{-1}(\mathcal{F}(0))$. Conversely, let $x_{0} \in \operatorname{ker}(A) \cap C^{-1}(\mathcal{F}(0))$, i.e., $A x_{0}=$ $0, C x_{0} \in \mathcal{F}(0)$. Consequently, $0 \in A x_{0}-B(\mathcal{F}+D)^{-1}\left(C x_{0}\right)$, i.e. $x_{0} \in \mathcal{W}$. Hence, $\operatorname{ker}(A) \cap C^{-1}(\mathcal{F}(0)) \subset \mathcal{W}$. In conclusion, $\mathcal{W}=\operatorname{ker}(A) \cap C^{-1}(\mathcal{F}(0))$.

iv) If there exists a negative definite matrix $Q$ such that $P=A^{T} Q$, then for each $x_{0} \in \mathcal{A}$, we have

$$
\lim _{t \rightarrow+\infty} d\left(x\left(t ; x_{0}\right), \mathcal{W}\right)=0 .
$$

In fact, $\langle P A x, x\rangle=0$ implies $\langle Q A x, A x\rangle=0$ and thus $A x=0$, which permits us to deduce that $\mathcal{W}=\mathcal{P}$, i.e. the set of equilibria is attractive. 


\section{Robust Stability of Set-valued Lur'e Dynamical Systems}

Stability of disturbed set-valued Lur'e dynamical systems in finite-dimensional spaces $\left(\mathcal{H}_{1}=\mathbb{R}^{n}\right.$ and $\left.\mathcal{H}_{2}=\mathbb{R}^{m}\right)$ is analysed in this section. We present some conditions of uncertain deviations such that the stability is preserved. The system $(\mathcal{S})$ is a model for certain real system in practice. Generally, due to the uncertainty or measured errors, the real system is different from $(\mathcal{S})$ by some uncertainties and governed by

$$
\left\{\begin{array}{l}
\dot{\bar{x}}(t)=\bar{A} \bar{x}(t)+\bar{B} \bar{\lambda}(t) \text { a.e. } t \in[0,+\infty) \\
\bar{y}(t)=C \bar{x}(t)+D \bar{\lambda}(t) \\
\bar{y}(t) \in \mathcal{F}(-\bar{\lambda}(t)), t \geq 0 \\
\bar{x}(0)=x_{0}
\end{array}\right.
$$

where $\bar{A}=A+\delta_{A}, \bar{B}=B+\delta_{B}$ with unknown deviations $\delta_{A}, \delta_{B}$. By the previous works developed in Section 4 , the passivity of a system may imply the stability of the origin. Our problem is to find conditions for $\delta_{A}, \delta_{B}$ such that the disturbed system (34) is still passive. Then the stability of (34) is maintained.

Lemma 3 Suppose that there exists a matrix $P=P^{T}>0$ such that

$$
\left(\begin{array}{cc}
P A+A^{T} P & P B-C^{T} \\
B^{T} P-C & -\left(D+D^{T}\right)
\end{array}\right)+\left(\begin{array}{cc}
P \delta_{A}+\delta_{A}^{T} P & P \delta_{B} \\
\delta_{B}^{T} P & 0_{m}
\end{array}\right) \leq 0
$$

then $(\bar{A}, \bar{B}, C, D)$ is passive.

Proof It is a direct consequence from Definition 2 of passivity.

Let

$$
Z=\overbrace{\left(\begin{array}{cc}
P A+A^{T} P & P B-C^{T} \\
B^{T} P-C & -\left(D+D^{T}\right)
\end{array}\right)}^{Z_{c}}+\overbrace{\left(\begin{array}{cc}
P \delta_{A}+\delta_{A}^{T} P & P \delta_{B} \\
\delta_{B}^{T} P & 0_{m}
\end{array}\right)}^{Z_{d}} .
$$

Denote $Z_{c}$ and $Z_{d}$ the constant part and disturbed part of $Z$ respectively then $Z=Z_{c}+Z_{d}$. Since $Z_{c}$ is symmetric, there exist an orthogonal matrix $\mathcal{O}$ and a diagonal matrix $\mathcal{D}$ such that $Z_{c}=\mathcal{O}^{T} \mathcal{D O}$.

Lemma 4 If $\mathcal{D}+\mathcal{O} Z_{d} \mathcal{O}^{T} \leq 0$ then $(\bar{A}, \bar{B}, C, D)$ is passive.

Proof Under the assumption above, for all $x \in \mathbb{R}^{n+m}$, we have

$$
\langle Z x, x\rangle=\left\langle\left(\mathcal{O}^{T} \mathcal{D} \mathcal{O}+Z_{d}\right) x, x\right\rangle=\left\langle\left(\mathcal{D}+\mathcal{O} Z_{d} \mathcal{O}^{T}\right) \mathcal{O} x, \mathcal{O} x\right\rangle \leq 0
$$

Hence $Z$ is negative semi definite. 
Remark 8 The condition in Lemma 4 is considered as a robustness measure since it provides some bounds of the deviations such that the disturbed system remains stable. For example, if

$$
\left(\mathcal{O} Z_{d} \mathcal{O}^{T}\right)_{i i} \leq-\mathcal{D}_{i i} \text { and }\left(\mathcal{O} Z_{d} \mathcal{O}^{T}\right)_{i j}=0, i \neq j,
$$

then the condition in Lemma 4 holds.

Proposition 3 Let Assumption 3 hold and suppose that the condition in Lemma 4 is satisfied. Then origin of the disturbed system (34) is stable.

In the next part, let us give another sufficient condition for the robust stability of the system (34) by using the S-procedure [21]. We recall the following lemma.

Lemma 5 [21] Let $T_{0}, T_{1}, \ldots, T_{p}$ be $n \times n$ symmetric matrices. The following condition

$$
\xi^{T} T_{0} \xi \leq 0 \text { for all } \xi \in \cap_{i=1}^{p}\left\{x \in \mathbb{R}^{n}: x^{T} T_{i} x \leq 0\right\}
$$

holds if there exist $\tau_{1} \geq 0, \tau_{2} \geq 0, \ldots, \tau_{p} \geq 0$ satisfying $T_{0}-\sum_{i=1}^{p} \tau_{i} T_{i} \leq 0$.

Here the unknown deviations are supposed to be in the following form

$$
\left[\delta_{A}(t) \quad \delta_{B}(t)\right]=F(t)\left[\begin{array}{ll}
E_{a} & E_{b}
\end{array}\right]
$$

where $E_{a}, E_{b}$ are constant matrix and $F(t)$ is time-varying matrix satisfying

$$
\|F(t)\| \leq 1 \text {. }
$$

Theorem 8 Let Assumption 3 hold. The origin of the disturbed system (34) is stable if there exist $P=P^{T}>0, v_{1} \geq 0, v_{2} \geq 0$ such that the following linear matrix inequality (LMI)

$$
\left(\begin{array}{cccc}
A^{T} P+P A+v_{1} E_{a}^{T} E_{a} & P B-C^{T} & P & P \\
B^{T} P-C & -\left(D+D^{T}\right)+v_{2} E_{b}^{T} E_{b} & 0 & 0 \\
P & 0 & -v_{1} I & 0 \\
P & 0 & 0 & -v_{2} I
\end{array}\right) \leq 0
$$

is solvable.

Proof Let

$$
u=\delta_{A} x=F(t) E_{a} x, v=\delta_{B} \lambda=F(t) E_{b} \lambda .
$$

We have

$$
\begin{aligned}
\left.\dot{V}\right|_{(34)}= & \langle P \bar{A} x, x\rangle+\langle P \bar{B} \lambda, x\rangle=\langle P A x, x\rangle+\langle P B \lambda, x\rangle+\langle u, P x\rangle+\langle v, P x\rangle \\
\leq & \langle P A x, x\rangle+\langle P B \lambda, x\rangle+\langle u, P x\rangle+\langle v, P x\rangle-\langle C x+D \lambda, \lambda\rangle \\
& (\text { note that }\langle C x+D \lambda, \lambda\rangle \leq 0 \text { since } C x+D \lambda \in \mathcal{F}(-\lambda)) \\
\leq & \langle P A x, x\rangle+\left\langle\left(P B-C^{T}\right) \lambda, x\right\rangle-\langle D \lambda, \lambda\rangle+\langle u, P x\rangle+\langle v, P x\rangle .
\end{aligned}
$$

Since $\|F(t)\| \leq 1$, we have

$$
u^{T} u \leq x^{T} E_{a}^{T} E_{a} x \text { and } v^{T} v \leq \lambda^{T} E_{b}^{T} E_{b} \lambda .
$$


Applying the S-procedure with

$$
\begin{aligned}
& T_{0}=\left(\begin{array}{cccc}
A^{T} P+P A & P B-C^{T} & P & P \\
B^{T} P-C & -\left(D+D^{T}\right) & 0 & 0 \\
P & 0 & 0 & 0 \\
P & 0 & 0 & 0
\end{array}\right), \\
& T_{1}=\left(\begin{array}{cccc}
-E_{a}^{T} E_{a} & 0 & 0 & 0 \\
0 & 0 & 0 & 0 \\
0 & 0 & I & 0 \\
0 & 0 & 0 & 0
\end{array}\right), T_{2}=\left(\begin{array}{cccc}
0 & 0 & 0 & 0 \\
0 & -E_{b}^{T} E_{b} & 0 & 0 \\
0 & 0 & 0 & 0 \\
0 & 0 & 0 & I
\end{array}\right) \text { and } \xi=\left(\begin{array}{c}
x \\
\lambda \\
u \\
v
\end{array}\right) .
\end{aligned}
$$

It implies that if the matrix inequality (35) is solvable, then $\left.\dot{V}\right|_{(34)} \leq 0$. Thus, the origin of the perturbed system is stable.

Remark 9 Theorem 8 allows us to check stability of the perturbed system by solving LMIs. There are many efficient numerical algorithms to solve LMIs, for example the interior-point methods [21].

\section{Some Illustrative Examples in Finite and Infinite Dimensional Spaces}

Here we provide two examples in electrical circuits (finite dimension) and one example in partial differential equation (infinite dimension) to illustrate the theoretical developments in previous sections. For the ampere-volt characteristics of some familiar electrical devices (resistors, ideal diodes, diacs, silicon controller rectifiers...), see [4, 22] for references. In particular, the voltage-current characteristic function $f_{\text {diac }}$ of a diac is upper semi-continuous on $\mathbb{R}$ with convex, compact values (Fig. 2). The mapping is set-valued at 0 and single-valued differentiable with bounded derivative in $\mathbb{R} \backslash\{0\}$ by some real number $k>0$. Then it is easy to see that $f_{\text {diac }}+k I$ is monotone.

Fig. 2 A typical voltage-current characteristics of a diac

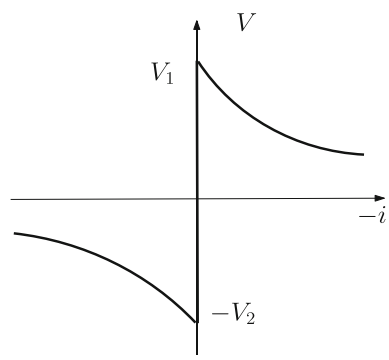


Example 1 Consider the circuit in Fig. 3 with a resistor $R>0$, a capacitor $C_{0}>0$, an inductor $L>0$ and a diac. Let $x_{1}$ be the charge on the capacitors and $x_{2}$ be the current through the inductors. Using Kirchhoff's circuit laws (see also [12]), we have

$$
\left(\begin{array}{c}
\dot{x}_{1} \\
\dot{x}_{2}
\end{array}\right)=\overbrace{\left(\begin{array}{cc}
0 & -1 \\
\frac{1}{L C_{0}} & 0
\end{array}\right)}^{A}\left(\begin{array}{c}
x_{1} \\
x_{2}
\end{array}\right)+\overbrace{\left(\begin{array}{l}
1 \\
0
\end{array}\right)}^{B} \lambda,
$$

where

$$
y=\overbrace{\left(\begin{array}{ll}
\frac{1}{C_{0}} & 0
\end{array}\right)}^{C}\left(\begin{array}{c}
x_{1} \\
x_{2}
\end{array}\right)+\overbrace{R}^{D} \lambda \text { and } y \in f_{\text {diac }}(-\lambda) .
$$

It is not difficult to verify that if $R \geq k$ ( $k$ is defined at the beginning of this section), then all the assumptions of Corollary 1 are satisfied with

$$
P=\left(\begin{array}{cc}
\frac{1}{C_{0}} & 0 \\
0 & L
\end{array}\right) \text {. }
$$

Thus the existence and uniqueness of solutions on $[0,+\infty)$ are ensured. In addition

$$
P B=C^{T} \text { and } P A=\left(\begin{array}{cc}
0 & -\frac{1}{C_{0}} \\
\frac{1}{C_{0}} & 0
\end{array}\right)
$$

is positive semidefinite and $0 \in f_{\text {diac }}(0)$. By Theorem 2 , the origin is stable. Note that all the assumptions of Theorem 7 also holds and we can compute the set $\mathcal{P}=C_{0} f_{\text {diac }}(0) \times \mathbb{R}$ which is defined in (33). Thus, one can obtain more information about $x_{1}(\cdot)$ that

$$
\lim _{t \rightarrow+\infty} d\left(x_{1}(t), C_{0} f_{\text {diac }}(0)\right)=0 .
$$

Let us consider the perturbed system of (36) by replacing $A, B$ by $A+\delta_{A}, B+\delta_{B}$ respectively and find certain condition such that the origin is still stable. Thanks to Lemma 4 , with some simple calculations, we have the following sufficient condition

$$
\left(\begin{array}{cc}
0_{2 \times 2} & 0_{2 \times 1} \\
0_{1 \times 2} & 2 R
\end{array}\right) \geq\left(\begin{array}{cc}
P \delta_{A}+\delta_{A}^{T} P & P \delta_{B} \\
\delta_{B}^{T} P & 0
\end{array}\right) \Leftrightarrow\left(\begin{array}{cc}
P \delta_{A}+\delta_{A}^{T} P & P \delta_{B} \\
\delta_{B}^{T} P & -2 R
\end{array}\right) \leq 0 .
$$

For example, if $\delta_{A}(1,1) \leq 0, \delta_{A}(2,2) \leq 0$ and $\delta_{A}(1,2)=\delta_{A}(2,1)=0, \delta_{B}=0_{2 \times 1}$ then the origin's stability of the perturbed system is maintained.

Fig. 3 RLC circuit with a diac

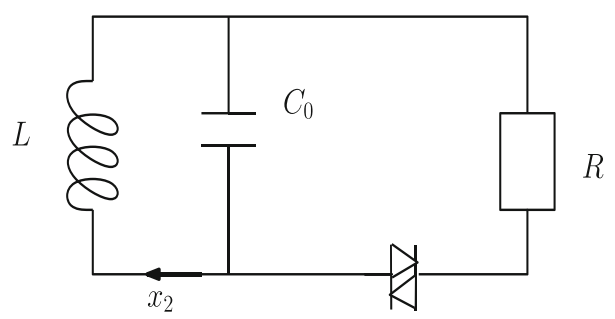


Example 2 Let us consider the four-diode bridge full wave rectifier in Fig. 4 with a resistor $R>0$, a capacitor $C_{0}>0$, an inductor $L>0$ and four ideal diodes (see [12] for reference). Using Kirchhoff's circuit laws, one obtains

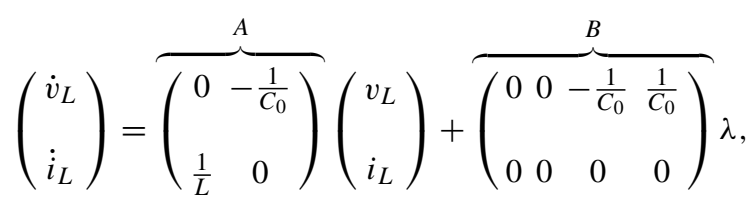

where

$$
\lambda=\left(\begin{array}{c}
-v_{\mathrm{DR} 1} \\
-v_{\mathrm{DF} 2} \\
i_{\mathrm{DF} 1} \\
i_{\mathrm{DR} 2}
\end{array}\right), y=\left(\begin{array}{c}
i_{\mathrm{DR} 1} \\
i_{\mathrm{DF} 2} \\
-v_{\mathrm{DF} 1} \\
-v_{\mathrm{DR} 2}
\end{array}\right), 0 \leq \lambda_{i} \perp y_{i} \geq 0 \text { for } i=1,2,3,4
$$

and

$$
y=\overbrace{\left(\begin{array}{cc}
0 & 0 \\
0 & 0 \\
-1 & 0 \\
1 & 0
\end{array}\right)}^{C}\left(\begin{array}{c}
v_{L} \\
i_{L}
\end{array}\right)+\overbrace{\left(\begin{array}{cccc}
\frac{1}{R} & \frac{1}{R} & -1 & 0 \\
\frac{1}{R} & \frac{1}{R} & 0 & -1 \\
1 & 0 & 0 & 0 \\
0 & 1 & 0 & 0
\end{array}\right)}^{D} \lambda .
$$

Note that $D$ is positive semidefinite but not positive definite. In this case, the maximal monotone operator $\mathcal{F}: \mathbb{R}^{4} \rightrightarrows \mathbb{R}^{4}$ is defined by $x=\left(\begin{array}{llll}x_{1} & x_{2} & x_{3} & x_{4}\end{array}\right)^{T} \mapsto \mathcal{F}(x)=$ $\left(N_{\mathbb{R}_{-}}\left(x_{1}\right) N_{\mathbb{R}_{-}}\left(x_{2}\right) N_{\mathbb{R}_{-}}\left(x_{3}\right) N_{\mathbb{R}_{-}}\left(x_{4}\right)\right)^{T}$ where $N$ denotes the normal cone. Then all the assumptions of Corollary 1 hold with

$$
P=\left(\begin{array}{cc}
C_{0} & 0 \\
& \\
0 & L
\end{array}\right),
$$

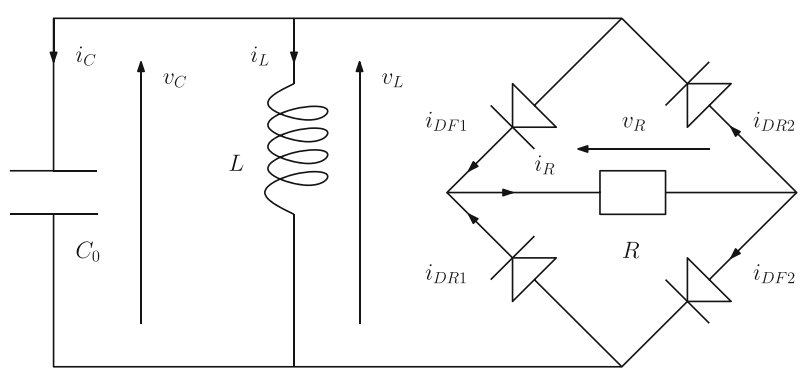

Fig. 4 A four-diode bridge wave rectifier 
which assures the existence and uniqueness of solutions on $[0,+\infty)$. Again, we have

$$
P B=C^{T} \text { and } P A=\left(\begin{array}{cc}
0 & -1 \\
1 & 0
\end{array}\right)
$$

is positive semidefinite and $0 \in \mathcal{F}(0)$. By Theorem 2 the origin is stable. Similarly, the origin of the disturbed system of (38) is stable if

$$
\left(\begin{array}{cc}
0_{2 \times 2} & 0_{2 \times 4} \\
0_{4 \times 2} & D+D^{T}
\end{array}\right) \geq\left(\begin{array}{cc}
P \delta_{A}+\delta_{A}^{T} P & P \delta_{B} \\
\delta_{B}^{T} P & 0_{4 \times 4}
\end{array}\right),
$$

or equivalently,

$$
\left(\begin{array}{cc}
0_{5 \times 5} & 0 \\
0 & \frac{4}{R}
\end{array}\right) \geq V\left(\begin{array}{cc}
P \delta_{A}+\delta_{A}^{T} P & P \delta_{B} \\
\delta_{B}^{T} P & 0_{4 \times 4}
\end{array}\right) V^{T},
$$

where $V$ is the orthogonal matrix defined by

$$
V=\left(\begin{array}{cccccc}
1 & 0 & 0 & 0 & 0 & 0 \\
0 & 1 & 0 & 0 & 0 & 0 \\
0 & 0 & -\frac{1}{\sqrt{2}} & 0 & 0 & \frac{1}{\sqrt{2}} \\
0 & 0 & \frac{1}{\sqrt{2}} & 0 & 0 & \frac{1}{\sqrt{2}} \\
0 & 0 & 0 & 1 & 0 & 0 \\
0 & 0 & 0 & 0 & 1 & 0
\end{array}\right) .
$$

Example 3 Let $H_{1}=H_{2}=L^{2}(0,1)$ and $\left(e_{1}, e_{2}, \ldots, e_{n}, \ldots\right)$ be an orthonormal basis of $L^{2}(0,1)$. Fix some positive integer $n$. Define the operators $A, B, C, D: L^{2}(0,1) \rightarrow$ $L^{2}(0,1)$ as follows: $-A=B=I$, the identity operator; $C e_{i}=0$ for $1 \leq i \leq n, C e_{i}=e_{i}$ for $i \geq n+1$; and $D e_{i}=e_{i}$ for $1 \leq i \leq n, C e_{i}=0$ for $i \geq n+1$. It is easy to verify that $A, B, C, D$ are symmetric linear bounded and $\left(A+\frac{3}{4} I, B, C, D\right)$ is passive. Furthermore $D$ is monotone but not positive definite and there is no symmetric positive definite $P \in$ $\mathcal{L}\left(L^{2}(0,1)\right)$ such that $P B=C^{T}$. Define $\mathcal{F}: L^{2}(0,1) \rightrightarrows L^{2}(0,1)$ by $\mathcal{F}=\partial\|\cdot\|$, then $\mathcal{F}$ is maximal monotone. Consider the following partial differential equation

$$
\frac{\partial \tilde{x}}{\partial t}(l, t)=A \tilde{x}(l, t)+B \tilde{\lambda}(l, t), \tilde{x}(l, 0)=\tilde{x}_{0}(l), \text { a.e. } 0 \leq l \leq 1, t \geq 0,
$$

with observation

$$
\tilde{y}(l, t)=C \tilde{x}(l, t)+D \tilde{\lambda}(l, t) \in \partial\|\cdot\|(\tilde{\lambda}(l, t)) .
$$

Let $x(t)=\tilde{x}(\cdot, t), \lambda(t)=\tilde{\lambda}(\cdot, t), y(t)=\tilde{y}(\cdot, t)$, one obtains the system in the form $(\mathcal{S})$ in infinite dimensional Hilbert space $L^{2}(0,1)$. Then all the assumptions of Theorem 1 are satisfies and hence there exists a unique Lipschitz continuous solution $x(\cdot)$ defined on $\mathbb{R}_{+}$. Furthermore, Assumption 3 is satisfied and $(A, B, C, D)$ is strictly passive. It follows that the origin is globally exponentially stable by using Theorem 3 . 


\section{Conclusion}

In this paper, the well-posedness and stability analysis of set-valued Lur'e dynamical systems with nonzero feedthrough operator $D$ in infinite-dimensional spaces have been thoroughly studied, under the so-called passivity condition. This work improves and extends in different ways the recent results obtained in $[2,6,7]$. The robust stability of the systems is investigated in the finite-dimensional case. We also provide examples in nonregular electrical circuits as well as in partial differential equations to illustrate the theoretical results. For upcoming works, it would be interesting to study deeply the role played by the passivity condition within the theory of maximal monotone operators; that is, to find a general framework where this condition ensures the maximal monotonicity of the composition of monotone operators. An other research topic will be the consideration of Lur'e dynamical systems with general initial condition, not necessarily a point in the domain of the involved operator.

Acknowledgments The research of the second author is supported by Fondecyt Regular Project 1151003 and the third author is supported by Fondecyt Project 3150332.

\section{References}

1. Liberzon, M.R.: Essays on the absolute stability theory. Autom. Remote. Control. 67(10), 1610-1644 (2006)

2. Brogliato, B., Goeleven, D.: Existence, uniqueness of solutions and stability of nonsmooth multivalued Lur'e dynamical systems. J. Convex Anal. 20(3), 881-900 (2013)

3. Brogliato, B., Lozano, R., Maschke, B., Egeland, O. Dissipative systems analysis and control, 2nd Edition. Springer, London (2007)

4. Adly, S., Le, B.K.: Stability and invariance results for a class of non-monotone set-valued Lur'e dynamical systems. Appl. Anal. 93(5), 1087-1105 (2014)

5. Brogliato, B.: Absolute stability and the Lagrange-Dirichlet theorem with monotone multivalued mappings. Syst. Control Lett. 51(5), 343-353 (2004)

6. Brogliato, B., Goeleven, D.: Well-posedness, stability and invariance results for a class of multivalued Lur'e dynamical systems. Nonlinear Anal. Theory Methods Appl. 74, 195-212 (2011)

7. Camlibel, M.K., Schumacher, J.M.: Linear passive systems and maximal monotone mappings. to appear in Mathematical Programming

8. Cojocaru, M.G., Daniele, P., Nagurney, A.: Projected dynamical systems and evolutionary variational inequalities via Hilbert spaces and applications. JOTA 127(3), 549-563 (2005)

9. Grabowski, P., Callier, F.M.: Lur'e feedback systems with both unbounded control and observation: Well-posedness and stability using nonlinear semigroups. Nonlinear Anal. 74, 3065-3085 (2011)

10. Gwinner, J., On differential variational inequalities and projected dynamical systems - equivalence and a stability result. Discrete and Continuous Dynamical Systems. issue special, pp. 467-476 (2007)

11. Gwinner, J.: On a new class of differential variational inequalities and a stability result. Math. Prog. 139(1-2), 205-221 (2013)

12. Acary, V., Brogliato, B.: Numerical methods for nonsmooth dynamical systems. Applications in Mechanics and Electronics, p. 35. Springer, LNACM (2008)

13. Miyagi, H., Yamashita, K.: Robust stability of Lure systems with multiple nonlinearities. IEEE Trans. on Auto. Cont. 37(6) (1992)

14. Rockafellar, R.T., Wets, R.J.B.: Variational analysis, vol. 317. Springer, Grundlehren der Mathematischen Wissenschaften (1998)

15. Aubin, J.P., Cellina, A.: Differential inclusions. Set-Valued Maps and Viability Theory. Spinger, Berlin (1984)

16. Brezis, H.: Opérateurs Maximaux Monotones et Semi-groupes de Contractions dans les Espaces de Hilbert. Math. Studies 5, North-Holland American Elsevier (1973)

17. Mordukhovich, B.S.: Variational analysis and generalized differentiation I \& II. Springer

18. Adly, S., Goeleven, D.: A stability theory for second-order nonsmooth dynamical systems with application to friction problems. J. Math. Pures et Appliquées 83(1), 17-51 (2004) 
19. Adly, S., Brogliato, B., Le, B.K.: Well-posedness, robustness and stability analysis of a set- valued controller for Lagrangian systems. SIAM J. Control Optim. 51(2), 1592-1614 (2013)

20. Adly, S., Hantoute, A., Thera, M.: Nonsmooth Lyapunov pairs for infinite-dimensional first-order differential inclusions. Nonlinear Anal. Theory Methods Appl. 75(3), 985-1008 (2012)

21. Boyd, S., Ghaoui, L., Feron, E., Balakrishnan, V.: Linear matrix inequalities in system and control theory. Studies in Applied Mathematics, vol. 15. SIAM, Philadelphia (1994)

22. Addi, K., Adly, S., Brogliato, B., Goeleven, D.: A method using the approach of Moreau and Panagiotopoulos for the mathematical formulation of nonregular circuits in electronics. Nonlinear Anal. Hybrid Syst. 1(1), 30-43 (2007) 